\title{
A CHECKLIST OF PTERIDOPHYTES COLLECTED ON CHAM CHU MOUNTAINS AND ADJACENT SITES
}

\author{
Phan Ke Loc ${ }^{1, *}$, Vu Thuy Duong ${ }^{2}$, Nguyen Cong Toan ${ }^{2}$,Pham Van The ${ }^{3}$, Trinh Ngoc Bon ${ }^{4}$ \\ ${ }^{1}$ Department of Botany, Faculty of Biology, VNU University of Science, Ha Noi, Vietnam \\ ${ }^{2}$ VNU University of Science, Biological Museum, The herbarium HNU, Ha Noi, Vietnam \\ ${ }^{3}$ Department for Management of Science and Technology Development and Faculty of Applied \\ Sciences, Ton Duc Thang University, Ho Chi Minh City, Vietnam \\ ${ }^{4}$ Department of Silviculture Foundation and Forest Phytodiversity, Silviculture Research \\ Institute, Vietnamese Academy of Forest Sciences, Ha Noi, Vietnam
}

Received 19 October 2019, accepted 23 December 2019

\section{ABTRACT}

Located in Tuyen Quang and Ha Giang Provinces, the Cham Chu Nature reserve (NR) has diverse plant ecosystem types, and its the flora is rich in species. However, the pteridophyte flora of Tuyen Quang and Ha Giang Provinces has not been so far generally studied. This study presented results of three biotic surveys conducted on Cham Chu mountains and adjacent sites surveys in 2018-2019. A total of 123 plant specimens gathered and analyzed. With 84 plant species of 43 genera belonging to 23 families recorded. The family Polypodiaceae is the richest with 8 genera and 12 species. Two genera, Tectaria and Pteris, are rich in species, with 9 and 8 species, respectively. A checklist of species was also provided.

Keywords: Polypodiaceae, checklist, diversity, Cham Chu Nature Reserve.

Citation: Phan Ke Loc, Vu Thuy Duong, Nguyen Cong Toan, Pham Van The, Trinh Ngoc Bon, 2019. A checklist of pteridophytes collected on Cham Chu mountains and adjacent sites. Academia Journal of Biology, 41(4): 75-89. https://doi.org/10.15625/2615-9023/v41n4.14639.

*Corresponding author email: pkeloc@yahoo.com

C2019 Vietnam Academy of Science and Technology (VAST) 


\section{INTRODUCTION}

Recently, the pteridophyte flora of Tuyen Quang and Ha Giang Provinces is not studied yet (Tardieu-Blot \& Christensen, 19391951). Therefore The NEF Bio-ecological Nature Conservation Project in Mountainous Region of North Vietnam were handed over us to study its pteridophyte flora in The Cham Chu Nature Reserve and adjacent sites.

\section{MATERIALS AND METHODS}

All studied sites are situated in less than $1000 \mathrm{~m}$ a.s.l., in lowland belts with limestone and/or sandstone--shale. Pteridophytes are mostly light demanding plants, growing on edges of forest, in scrubs or grasslands, perennial terrestrial, epiphytic and lithophytic. Three fieldworks were set up in the $1^{\text {st }}$ step (2018-2019): 1.Tuyen Quang Prov., Ham Yen Distr., foot of Cham Chu mountain, including Doi Lo (Oct 2018), collected 36 collecting numbers by Phan Ke Loc, Pham Van The and Vu Thuy Duong (with symbol $T L$ ); 2. Tuyen Quang Prov., Ham Yen Distr., Yen Thuan Comm, Cao Duong Vill., near H'Mông subvill., (Apr 2019), collected 57 collecting numbers by Phan Ke Loc, Vu Thuy Duong and Pham Van The (symbol LT) and 3. Ha Giang Prov., Bac Me Distr., Minh Ngoc Comm., Lung Cang Vill. (Jul 2019), collected 64 collecting numbers by $\mathrm{Vu}$ Thuy Duong, Thao Van Chien and Phan Ke Loc (symbol $D L-B M)$. All together 153 numbers were collected. Main sources are used to identify scientific names of taxa (DeVol C. E. et al., 1994; Parris B. S. et al., 2013; Parris B. S. et al., 2010; Phạmhoàng Hộ, 1991; Phan Ke Loc, 2010; Tagawa M. \& Iwatsuki K., 1979; Tagawa M. \& Iwatsuki K., 1985; Tagawa M. \& Iwatsuki K., 1988; Tagawa M. \& Iwatsuki K., 1989; Tardieu-Blot M. L. \& Christensen C., 1939-1951; Wu Z. Y. et al., 2013a; Wu Z. Y. et al., 2013b).

\section{RESULTS AND DISCUSSION}

The collected numbers belong to 84 species in 43 genera and 23 families. They are arranged in descending sequence of principal ranks, namely Plant Kingdom, Tracheophytes (exclus seed plants) Phylum/Pteridophytes with Class, Order, Family, Genus and Species (Shenzhen Code 2018, 2018). PPG I (2016) is adopted in this paper (PPG I (2016), 2016). Genera in family, and species in genus are arranged in alphabetical order.

\section{System of classification}

CLASS Lycopodiopsida

Order Lycopodiales

Family Lycopodiaceae

Genus Phlegmariurus, three species: $P$. fargesii (Herter) Ching, P.phlegmaria (L.) Roth and P.squarrosus (G.Forst.) Á.Löve \& D.Löve

Order Selaginellales

Family Selaginellaceae

Genus Selaginella, five species: $S$. Bodinieri Hieron., $S$. decipiens Warb. S.doederleinii Hieron., S. indica (Milde) R.M.Tryon and S.willdenowii (Desv.) Baker

CLASS Polypodiopsida

Order Equisetales

Family Equisetaceae, one genus Equisetum with one species: $E$. ramosissimum Desf.

Order Psilotales

Family Psilotaceae, one genus Psilotum with one species: P. nudum (L.) P.Beauv.

Order Marattiales

Family Marattiaceae, one genus Angiopteris with one species: $A$. confertinervia Ching ex C.Chr.

Order Gleicheniales

Family Gleicheniaceae, one genus Dicranopteris, one species: $D$. pedata (Houtt.) Nakaike

Order Schizaeales

Family Lygodiaceae, one genus Lygodium, two species: L. circinnatum (Burm.f.) Sw. and L. flexuosum (L.) Sw.

Order Cyatheales

Family Cibotiaceae, one genus with one species: C. barometz(L.) J.Sm. 
Family Cyatheaceae, one genus: Alsophila with two species: A. gigantea Wall. ex Hook. and $A$. podophylla Hook.

\section{Order Polypodiales}

Family Lindsaeaceae, two genera, Lindsaea with two species: L. ensifolia Sw. and L. orbiculata (Lam.) Mett. ex Kuhn and Odontosoria with one species: $O$. chinensis (L.) J.Sm.

Family Pteridaceae, five genera, Adiantum with one species: A. caudatum L.; Antrophyum with three species: A. callifolium Blume, A. obovatum Baker and A. vittarioides Baker; Haplopteris with two species: $H$. elongata (Sw.) E.H.Crane and $H$. flexuosa (Fée) E.H.Crane; Pityrogramma with one species: P. calomelanos (L.) Link; and Pteris with eight species: P.cretica L., P. decrescens H.Christ, $P$. ensiforme Burm.f., $P$. fauriei Hieron., $P$. plumbea H.Christ, $P$. semipinnata L., $P$. tripartita $\mathrm{Sw}$. and $P$. vittata $\mathrm{L}$.

Family Dennstaedtiaceae, two genera, Microlepia with one species: M. speluncae (L.) T.Moore; and Pteridium with one species: P. esculentum (G.Forst.) Cokaine

Family Aspleniaceae, two genera, Asplenium with three species: $A$. antrophyoides H.Christ, A. ensiforme Wall. ex Hook. \& Grev. and A. nidus L. and Hymenasplenium with one species: $H$. obliquissimum (Hayata) Sugimoto

Family Blechnaceae, one genus Blechnum with one species: $B$. orientale $\mathrm{L}$.

Family Athyriaceae, two genera: Athyrium with one species: A. mackinnonii (C.Hope) C.Chr.; and Diplazium with four species: $D$. donianum (Mett.) Tardieu, D. esculentum (Retz.) Sw., D. megaphyllum (Baker) H.Christ and D. stenochlamys C.Chr.

Family Thelypteridaceae, three genera: Christella with one species: $C$. balansae (Ching) Holttum; Cyclosorus with two species: C. parasiticus (L.) Farw. and $C$. truncatus (Poir.) Farw.; and Pronephrium with two species: $P$. hirsutum Ching ex Y.X.Lin and $P$. simplex var. trifoliata (Ching) Loc K. Phan, var. nov.
Family Dryopteridaceae, two genera, Bolbitis with two species: B. christensenii (Ching) Ching and B. heteroclita (C.Presl) Ching; and Dryopteris with one species: $D$. varia (L.) Kunze

Family Nephrolepidaceae, one genus Nephrolepis with 2 species: $N$. biserrata (Sw.) Schott and N. cordifolia (L.) C.Presl.

Family Lomariopsidaceae, one genus Lomariopsis with one species: L spectabilis (Kunze) Mett.

Family Tectariaceae, three genera : Arthropteris with one species: A. palisotii (Desv.) Alston; Pleocnemia with one species: $P$. winitii Holttum; and Tectaria with nine species: T. decurrens (C.Presl) Copel., T. devexa (Kunze ex Mett.) Copel., T. fuscipes (Wall. ex Bedd.) Chr., T. kusukusensis (Hayata) Lellinger, T. phaeocaulis (Rosenst.) C.Chr., T. quinquefida (Baker) Ching, T. sagenioides (Mett.) Christenh., T. simonsii (Baker) Ching and T. subsageniacea (H.Christ) Christenh.

Family Oleandraceae, one genus Oleandra with one species: O. wallichii (Hook.) C.Presl

Family Davalliaceae, one genus Davallia with two species: $D$. divaricata Blume and $D$. solida (G.Forst.) Sw.

Family Polypodiaceae, eight genera: Aglaomorpha with one species: A. coronans (Wall ex Mett.) Copel.; Drynaria with one species: D. bonii H.Christ; Lepidomicrosorium with one species: L. superficiale (Blume) L.Wang; Leptochilus with two species: $L$. ellipticus (Thunb.) Noot. and L. henryi (Baker) X.C.Zhang; Microsorum with two species:M. membranaceum (D.Don) Ching and $M$. punctatum (L.) Copel.; Neolepisorus with three species: N. minor W.M.Chu, N. ovatus (Wall. ex Bedd.) Ching and $N$. zippelii (Blume) L.Wang; Phymatosorus with one species: $P$. cuspidatus (D.Don) Pic-Serm.; and Pyrrosia with one species: $P$. porosa (C.Presl) Hovenkamp.

\section{Checklist of studied taxa}

Data of each taxa are presented in following order: name of family, name of 
genus and species, studied collecting number, locality including coordinate, habitat and habit.

Family Lycopodiaceae

Phlegmariurus fargesii (Herter) ChingDL-BM 046-Ha Giang, Bac Me, Minh Ngoc, $22^{\circ} 42^{\prime} 49^{\prime \prime}, 105^{\circ} 11^{\prime} 16$ ", $224 \mathrm{~m}$. Epiphytic and photophytic on tree trunks at stream banks of limestone mountains. Stem caespitose, 0.6$0.9 \mathrm{~m}$ long, pendulous, cordlike; trophophylls dense, lanceolate, ca. $1-1.4 \times 0.01-0.15 \mathrm{~cm}$, strongly arched, with the tips pointing toward stem. Cultivated as ornamental plant. Rare.

Phlegmariurus phlegmaria (L.) Roth-LT 17, LT 63-Tuyen Quang, Ham Yen, Yen Thuan, 22 $2^{\circ} 7^{\prime} 07^{\prime \prime}, \quad 104^{\circ} 59^{\prime} 30^{\prime \prime}, 608 \mathrm{~m}$. Perennial xerophytic epiphytic and photophytic, in axil of Livistona spp. leaves, sandstone mountains; pendulous, $0.4-0.6 \mathrm{~m}$ long, 4-6 times dichotomously branched; trophophylls hard, spreading, ovate-triangular, ca. 8-12 mm long, 3-4 $\mathrm{mm}$ wide at base, apex very pointed; strobili terminal on branches, linear, divided, 6-9 $\mathrm{cm}$ long, like horsetail plants. Common.

Phlegmariurus phlegmaria (L.) Roth- $D L$ $B M$ 066-Ha Giang, Bac Me, Minh Ngoc, 22043'13”, 105¹1'32”, 211 m. Mesophytic, epiphytic on tree trunk along stream banks; trophophylls soft, spreading, ovate-triangular, ca. 8-12 mm long, 3-4 $\mathrm{mm}$ wide at base, apex not pointed. Rare.

Phlegmariurus squarrosus (G.Forst.) Á.Löve \& D.Löve-DL-BM 068-Ha Giang, Bac Me, Minh Ngoc. Epiphytic and photophytic on tree trunks at stream banks of limestone mountains. Stem caespitose, 0.4-0.6 m long, pendulous; trophophylls dense, lanceolate, ca. $1.2-1,4 \times 0.01-0.15 \mathrm{~cm}$, attached at right angle; strobili terminal on branches. Cultivated as ornamental plant. Rare.

Selaginella bodinieri Hieron.-DL-BM 021Ha Giang, Bac Me, Minh Ngoc, 22 42'49”, $105^{\circ} 11^{\prime} 16^{\prime \prime}, 224 \mathrm{~m}$. Perennial terrestrial or epiphytic, erect from decumbent stem, 0.3-0.5 $\mathrm{m}$ with creeping rhizome and stolons; main stem pinnately branched, 6-10 pairs; strobili solitary, ca. $2.5-3.5 \mathrm{~cm}$ long. Rare.
Selaginella decipiens Warb-DL-BM 064Ha Giang, Bac Me, Minh Ngoc, 22 42'49”, $105^{\circ} 11^{\prime} 16^{\prime}, 224 \mathrm{~m}$. Ombrophylic epiphytic on small tree trunk along stream banks of limestone mountain. Upper portion erect, ca. 15-25 cm, with creeping stolons and rhizome; ca. $0.5-0.7 \times 0.3 \mathrm{~m}$; lamina frond triangular in outline, ca. $0.2-0.3 \times 0.1-0.15$ $\mathrm{m}$; primary leafy branches 6-7 pairs twice pinnately branches; strobili terminal, solitary. Rather common.

Selaginella doederleinii Hieron.-LT 37Tuyen Quang, Ham Yen, Yen Thuan, $22^{\circ} 17^{\prime} 29^{\prime \prime}, 104^{\circ} 58^{\prime} 58^{\prime \prime}, 659 \mathrm{~m}$. In mesophytic secondary scrubs, edge sandstone of cave. Plants prostrate then erect, $0.3-0.4 \mathrm{~m}$ tall; leafy portion including leaves ca. $1 \mathrm{~cm}$ wide; strobili solitary or in pairs or forked, ca. $4 \mathrm{x}$ $0.1-0.22 \mathrm{~cm}$. Occasionally common.

Selaginella indica (Milde) R.M.Tryon$D L-B M$ 036-Ha Giang, Bac Me, Minh Ngoc, $22^{\circ} 43^{\prime} 41^{\prime \prime}, 105^{\circ} 12^{\prime} 22^{\prime \prime}, 200 \mathrm{~m}$. Perennial photophytic and mesophytic, on vertical cliffs of limestone mountain, $0.2-0.3 \times 0.1-0.15 \mathrm{~m}$; rhizophores at intervals throughout length of main stem; strobili solitary, ca. $1.5-2 \mathrm{~cm}$. Very common.

Family Selaginellaceae

Selaginella willdenowii (Desv.) Baker-LT 31-Tuyen Quang, Ham Yen, Yen Thuan, $22^{\circ} 17^{\prime} 29^{\prime \prime}, 104^{\circ} 58^{\prime} 58^{\prime \prime}, 659 \mathrm{~m}$. In semi-open scrub, foot of limestone mountains. Perennial terrestrial mesophytic, creeping to $2 \mathrm{~m}$ or more long; main stem branched throughout; leaves metallic green. Rather common.

Family Equisetaceae

Equisetum ramosissimum Desf.-TL 009Tuyen Quang Prov., Ham Yen Distr., Phu Luu Comm., 22 12'37', 10503'14”, $152 \mathrm{~m}$. Perennial hydrophytic fern, in groups on open wet stream banks; areal stem monomorphic, 0.6-0.9 $\mathrm{m}$ tall, $2-3 \mathrm{~mm}$ in diam. at middle portion, not obviously branched, strobilus ellipsoid. Ocasionally common.

Equisetum ramosissimum Desf.-TL 56Tuyen Quang Prov., Ham Yen Distr., Phu Luu Comm., 22 $2^{\circ} 12^{\prime} 50^{\prime \prime}, 105^{\circ} 02^{\prime} 30^{\prime \prime}, 100$ m. In groups on open water current very slow; 
perennial hydrophytic fern; areal stem monomorphic, $1-1.2 \mathrm{~m}$ tall, $4-6 \mathrm{~mm}$ in diam. at middle portion, not obviously branched; strobilus ellipsoid, ca. 5-10 x 3-4 mm. Occasionally common.

Family Psilotaceae

Psilotum nudum (L.) P.Beauv.-DL-BM 051-Ha Giang, Bac Me, Minh Ngoc, $22^{\circ} 42^{\prime} 49^{\prime \prime}, 105^{\circ} 11^{\prime} 16^{\prime \prime}, 224 \mathrm{~m}$. Epiphytic on trunk and branches of trees on limestone stream banks. Not common.

\section{Family Marattiaceae}

Angiopteris confertinervia Ching ex C.Chr. \& Tardieu-TL 003, TL 011 \& $T L$ 017- Tuyen Quang, Ham Yen, Phu Luu, around $22^{\circ} 12^{\prime} 38^{\prime \prime}, 105^{\circ} 02^{\prime} 58^{\prime \prime}, 70-233 \mathrm{~m}$. Mainly in secondary mesophytic scrubs. Perennial terrestrial fern; frond $2.5-4 \mathrm{~m}$ long including stipe $1 \mathrm{~m}$; lamina bipinnate; pinnae ca. $0.4-0.8 \times 0.13-0.25 \mathrm{~m}$ with 14 20 pairs of pinnules; pinnules $5-17 \times 1.2$ $3.2 \mathrm{~cm}$, apices \pm long caudate; sori $2.5-3.5$ $\mathrm{mm}$, composed of 8-12 pairs of sporangia. Occasionally common.

Angiopteris confertinervia Ching ex C.Chr. \& Tardieu-DL-BM 003-Ha Giang, Bac Me, Minh Ngoc, 2243'17', 105¹1'33”, 210 $\mathrm{m}$. Habitat and habit same as TL 003, TL 011 \& TL 017. Common.

\section{Family Gleicheniaceae}

Dicranopteris pedata (Houtt.) NakaikeTL 021-Tuyen Quang, Ham Yen, Phu Luu, $22^{\circ} 12^{\prime} 38^{\prime \prime}, 105^{\circ} 02^{\prime} 57^{\prime \prime}, 140 \mathrm{~m}$. Clustered in secondary scrubs and grasslands of shale cliffs. Ferns 1-3 m tall; rachis 1-2 times dichotomously branched; ultimate pinnately linear, $0.2-0.3 \times 0.04-0.05 \mathrm{~m}$; lobes $30-50$ on each side, linear-lanceolate, $2-3 \times 0.3-0.4$ $\mathrm{cm}$, apex obtus. Occasionally common.

Family Lygodiaceae

Lygodium circinnatum (Burm.f.) Sw.-DLBM 019-Ha Giang, Bac Me, Minh Ngoc, 22043'41”, 105'12'22”, $200 \mathrm{~m}$. Growing commonly on secondary mesophytic scrubs on foot of mountains. Terrestrial perennial vines, to $15-20 \mathrm{~m}$ long; fertile pinnae usually sessile in pairs at end of ultimate branches; lamina \pm reduced and commonly 3-6 $\mathrm{mm}$ wide; sorophores $2-5 \mathrm{~mm}$, sessile. Common.

Lygodium flexuosum (L.) Sw.-TL 047aTuyen Quang, Ham Yen, Phu Luu, 22 ${ }^{\circ} 12^{\prime} 10^{\prime \prime}$, $105^{\circ} 02^{\prime} 40^{\prime \prime}, 170 \mathrm{~m}$. Scattered on secondary mesophytic shale scrubs. Perennial terrestrial vines, climbing on Livistona spp. and treelets; fronds 5-10 $\mathrm{m}$ long; sterile pinnae $3-10 \mathrm{~cm} \mathrm{x}$ 8-15 mm above; fertile pinnae smaller; sorophores 4-6 $\mathrm{mm}$ at apices of small triangular lobes; indusia glabrous. Common.

\section{Family Cibotiaceae}

Cibotium barometz (L.) J.Sm.-LT 18Tuyen Quang, Ham Yen, Phu Luu, 22 ${ }^{\circ} 17^{\prime} 30$ ”, $104^{\circ} 58^{\prime} 30^{\prime \prime}, 662 \mathrm{~m}$. In secondary grasslands and scrubs on sandstone-shale. Rhizome densely covered with soft, pale brownish, multicellular long hairs. Fronds 3-4 m tall; stipes thick, to $1 \mathrm{~m}$ or more long; lamina 2-3 $\mathrm{m}$ long, 2-pinnate-pinnatifid; pinnae 25-30 pairs; medial pinnae $0.6-0.8 \times 0.16-0.18 \mathrm{~m}$; medial pinnule $13 \times 1.5 \mathrm{~cm}$; sori usually $4-5$ each side of pinnule segments; indusia bivalvate. Rare.

Cibotium barometz (L.) J.Sm.-TL 046Tuyen Quang, Ham Yen, Phu Luu, $22^{\circ} 12^{\prime} 10^{\prime \prime}, 105^{\circ} 02^{\prime} 40^{\prime \prime}, 170 \mathrm{~m}$. Scattered on mesophytic secondary shale cliffs. Habit same as $L T$ 18. Rare.

Cibotium barometz (L.) J.Sm.-TL 55Tuyen Quang Prov., Ham Yen Distr., Phù Lưu Comm., 22 ${ }^{\circ} 12^{\prime} 50^{\prime \prime}, 105^{\circ} 02^{\prime} 30^{\prime}$ ', 125 m. Very young plant scattered on vertical cliffs of shale. Rhizome massive, ascending, densely covered with soft, pale brownish, multicellular long hairs. Rare.

Family Cyatheaceae

Alsophila gigantea Wall.ex Hook.-TTL 001, TL 15, TL 058 \& Thinh 001-Tuyen Quang, Ham Yen, Phu Luu, around $22^{\circ} 12^{\prime} 02^{\prime \prime}, 105^{\circ} 02^{\prime} 15$ ', 54-143 m. Scattered in secondary mesophytic shale cliffs. Trunk 2-4 $\mathrm{m}$ tall, $0.1-0.2 \mathrm{~m}$ in diam.; frond $2-3 \mathrm{~m}$ long, 2-pinnate-pinnatifid; base and adaxial side stipe with dense dark brown spreading scales ca. $1.5-2 \mathrm{~cm}$ long, $1.5-2.5 \mathrm{~mm}$ wide at base; lamina with 10-27 pairs pinnae, spreading, middle $0.4-0.5 \times 0.2 \mathrm{~m}$, pinnatifid 
to $1 / 2-2 / 3$ to costulae; pinnule segments ca. 14-18 pairs; sori orbicular. Common.

Alsophila podophylla Hook.-LT 20-Tuyen Quang, Ham Yen, Yen Thuan, 22 ${ }^{\circ} 17^{\prime} 29^{\prime \prime}$, $104^{\circ} 58^{\prime} 58^{\prime \prime}, 659 \mathrm{~m}$. In secondary sandstoneshale scrubs. Perennial terrestrial mesophytic fern; trunk 3-4 m tall; stipe to $1 \mathrm{~m}$, dark brown with setose; lamina 1-2 m, 2-pinnate; pinnae $7-18$ pairs, $0.35 \times 0.19 \mathrm{~m}$; pinnules 10-18 pairs; veinlets 4-5 pairs, basiscopic ones arising directly from base of pinna costa; sori orbicular. Not rare.

\section{Family Lindsaeaceae}

Lindsaea ensifolia Sw.-TL 047-Tuyen Quang, Ham Yen, Phu Luu, 22 $2^{\circ} 12^{\prime} 10^{\prime \prime}$, $105^{\circ} 02^{\prime} 40^{\prime \prime}, 170 \mathrm{~m}$. Scattered in secondary mesophytic scrubs on shale cliffs. Perennial terrestrial fern; rhizomes shortly creeping; stipes $0.1-0.25 \mathrm{~m}$; lamina 1 pinnate; pinnae 1-3 pairs, narrowly lanceolate, ca. 6-15 x 1.7-2.2 cm; sori marginal; indusia linear, continuous. Rare.

Lindsaea orbiculata (Lam.) Mett. ex Kuhn- TL 048-Tuyen Quang, Ham Yen, Phu Luu, 22 ${ }^{\circ} 12^{\prime} 10^{\prime \prime}, 105^{\circ} 02^{\prime} 40^{\prime \prime}, 170 \mathrm{~m}$. Scattered in secondary mesophytic scrubs on shale cliffs. Perennial terrestrial fern; rhizome shortly creeping; stipes $0.1-0.13 \mathrm{~m}$; lamina 0.3-0.4 x 0.02-0.07 m, 1-or 2-pinnate; pinnae 20-27 pairs, dimidiate, rhomboid, flabellate or orbicular; pinnae reduced towards apex; terminal pinna similar lateral ones; sori submarginal; indusia linear, usually continuous. Rare.

Odontosoria chinensis (L.) J.Sm.-LT 42Tuyen Quang, Ham Yen, Yen Thuan, $22^{\circ} 17^{\prime} 07^{\prime \prime}, 104^{\circ} 59^{\prime} 29^{\prime \prime}, 618 \mathrm{~m}$. In secondary mesophytic sandstone scrubs along roadsides. Perennial terrestrial fern; rhizome creeping, densely scaly; stipe $15-21 \mathrm{~cm}$ long; lamina oblong in outline, $0.2-0.3 \mathrm{x}$ 0.09-0.012 m, 3-or 4-pinnate, widest at middle; pinnae $10-15$ pairs, lanceolate, 3pinnate at base; sori terminal on 1 or uniting 2 or 3 vein ends. Rare.

\section{Family Pteridaceae}

Adiantum caudatum L.- DL-BM 032- Ha Giang, Bac Me, Minh Ngoc, 22³' 41", $105^{\circ} 12^{\prime} 22^{\prime}, 200 \mathrm{~m}$. In open vertical cliffs or rock crevices. Perennial terrestrial, lithophytic or epiphytic fern, $0.2-0.4 \mathrm{~m}$ tall; lamina 1pinnate, $12-37 \times 2-3 \mathrm{~cm}$, apex usually prolonged into a whiplike stolon rooting at tip to form new plantlet; pinnules $20-40$ pairs, \pm dimidiate, suboblong, $1-1.2 \times 0.5-0.8 \mathrm{~cm}$, base asymmetrical, lower margins entire, upper and outer margins deeply divided into lobes; sori 6-11 per pinna. Occasionally common.

Antrophyum callifolium Blume-TL 037Tuyen Quang, Ham Yen, Phu Luu, 22 ${ }^{\circ} 12^{\prime} 18^{\prime \prime}$, $105^{\circ} 02^{\prime} 38^{\prime \prime}, 212 \mathrm{~m}$. Epiphytic on small trees growing on secondary woodlands near stream banks. Perennial fern; rhizome short erect; fronds clustered; stipes $1-5 \mathrm{~cm}$; lamina obovate-lanceolate, $18-27 \times 2-4 \mathrm{~cm}$, widest above middle, base decurrent along appressed stipe; soral lines continuous. Common.

Antrophyum callifolium Blume-DL-BM 033-Ha Giang, Bac Me, Minh Ngoc, $22^{\circ} 43^{\prime} 41^{\prime \prime}, 105^{\circ} 12^{\prime} 22^{\prime \prime}, 200 \mathrm{~m}$. Habitat and habit same as TL 037 .

Antrophyum obovatum Baker-DL-BM 008-Ha Giang, Bac Me, Minh Ngoc, $22^{\circ} 42^{\prime} 49^{\prime \prime}, 105^{\circ} 11^{\prime} 16^{\prime \prime}, 224 \mathrm{~m}$. Growing as epiphytic in secondary woodlands. Rhizome short, erect; fronds clustered; stipe 1-4 cm, glabrous; lamina obovate-oblanceolate, 24-28 x 3-6 cm; widest above middle, base decurrent; soral lines superficial. Occasionally common.

Antrophyum vittarioides Baker-DL-BM 033B-Ha Giang, Bac Me, Minh Ngoc, $22^{\circ} 43^{\prime} 41^{\prime \prime}, 105^{\circ} 12^{\prime} 22^{\prime \prime}, 200 \mathrm{~m}$. Epiphytic on humid secondary scrubs. Rhizome short; fronds clustered; stipe 1-2 cm; lamina linearelliptic, $10-18 \times 0.5-0.7 \mathrm{~cm}$; soral lines linear, submarginal, one row on each side of costa, not immersed in grooves. Rare.

Haplopteris elongata (Sw.) E.H.CraneDL-BM 011-Ha Giang, Bac Me, Minh Ngoc, $22^{\circ} 44^{\prime} 55^{\prime \prime}, 105^{\circ} 11^{\prime} 36^{\prime \prime}, 210 \mathrm{~m}$. Scattered in mesophytic woodlands on foot of limestone. Epiphytic or lithophytic, grows in dense groups with other epiphytic ferns. Fronds clustered, pendulous, 1-2.3 m long, 0.5-2 $\mathrm{cm}$ wide, base gradually narrowed; soral 
line marginal, immersed in groove, open outward. Rare.

Haplopterisflexuosa (Fée) E.H.Crane-TL 050-Tuyen Quang, Ham Yen, Phu Luu, $22^{\circ} 12^{\prime} 50^{\prime \prime}, 105^{\circ} 02^{\prime} 30^{\prime \prime}, 125 \mathrm{~m}$. Epiphytic in leaf axil of Livistona spp. Rhizome thick; scales dark brown; fronds clustered; stipes slender; lamina linear, 12-45 x 0.4-0.6 cm, base long attenuate, margin revolute and covering sori. Very common.

Pityrogramma calomelanos (L.) Link-TL 041-Tuyen Quang Prov., Ham Yen Distr., Phu Luu, $22^{\circ} 12^{\prime} 10^{\prime \prime}, 105^{\circ} 02^{\prime} 40^{\prime \prime}, 152 \mathrm{~m}$. On secondary shale cliffs. Perennial terrestrial fern, to $0.8 \mathrm{~m}$ tall; stipe and axe of lamina purplish black, lustrous; lamina 2-pinnate, 18$32 \times 12-18 \mathrm{~cm}$, abaxially densely white farinose; pinnae 15-20 pairs; sori nearly confluent throughout abaxial surface. Rare.

Pteriscretica L.-LT 50a-Tuyen Quang, Ham Yen, Yen Thuan, 22 $2^{\circ} 17^{\prime} 07^{\prime \prime}$, 104'59'29”, 618 m. In shale-sandstone grasslands.Fronds dimorphic, $0.15-0.28$ x 0.05-0.08 m; stipe straw-colored; sterile fronds with stipes ca. $6-8 \mathrm{~cm}$ long; fertile fronds longer with stipe $10-15 \mathrm{~cm}$ long; pinnae 1-2 pairs, opposite, sessile; basal pinnae with 1 , rarely 2 pinnules; terminal pinna 3-lobed or simple, ca. 8-12 x 0.4-0.5 $\mathrm{cm}$, base decurrent. Rare.

Pteris decrescens H.Christ-DL-BM 040Ha Giang, Bac Me, Minh Ngoc, 22 43 '41", $105^{\circ} 12^{\prime} 22^{\prime \prime}, \quad 200 \mathrm{~m}$, In secondary wet grasslands on limestone slopes. Perennial fern, $0.7-0.9 \mathrm{~m}$ tall; fronds clustered;stipe brown, $25-30 \mathrm{~cm}$ long; lamina thin, glabrous, 2-pinnatipartite, oblong-lanceolate in outline, $0.5 \times 0.12-0.17 \mathrm{~m}$; lateral pinnae $9-11$ pairs, ca. $10-15 \times 2-2.6 \mathrm{~cm}$, apex narrowly linearcaudate, $3-5 \mathrm{~cm}$; segments more than $25-30$ pairs. Not common.

Pteris ensiformis Burm.f.-DL-BM 020Ha Giang, Bac Me, Minh Ngoc, 22 43'13", $105^{\circ} 11^{\prime} 32^{\prime}$, $211 \mathrm{~m}$. On acidic, wet scrubs and grasslands. Perennial terrestrial fern, ca. 0.3-0.5 m tall; fronds dimorphic; fertile specimens with stipe to $0.2-0.3 \mathrm{~m}$, lamina $20-42 \times 0.8-1 \mathrm{~cm}$, pinnate to bipinnate; terminal forked $12-22 \times 0.5 \mathrm{~cm}$. Common.
Pteris fauriei Hieron.-LT $27 \& L T$ 40Tuyen Quang, Ham Yen, Yen Thuan, around $22^{\circ} 17^{\prime} 30^{\prime \prime}, 104^{\circ} 58^{\prime} 58^{\prime \prime}, 618-645 \mathrm{~m}$. In wet sandstone-shale scrubs. Perennial terrestrial fern, $0.4-0.5 \mathrm{~m}$ tall; fronds clustered; stipe 17-32 cm; lamina 2-pinnatipartite, $0.3-0.5 \mathrm{x}$ 0.08-0.11 m; lateral pinnae 6-7 pairs; lowest basal pair with a basiscopic pinnule; upper pairs 8-12 x $0.18-0.23 \mathrm{~cm}$, with $15-22$ pairs of segments. Rather common.

Pteris fauriei Hieron.-DL-BM 030-Ha Giang, Bac Me, Minh Ngoc, 22'42'49", $105^{\circ} 11^{\prime} 16^{\prime \prime}, 224 \mathrm{~m}$. Habitat and habit same as LT 27 and LT 40.

Pteris plumbea H.Christ-LT 50-Tuyen Quang, Ham Yen, Yen Thuan, 22 $17^{\prime} 07^{\prime \prime}$, $104^{\circ} 59^{\prime} 29$ ”, $618 \mathrm{~m}$. In secondary wet shale grasslands. Plants 0.3-0.45 m tall, dimorphic; rhizome with dark brown scales; fronds clustered; stipes 4-angled, $0.25-0.35 \mathrm{~m}$ long; lamina of sterile fronds glabrous, $0.2-0.3 \mathrm{x}$ 0.1-0.15 m, 1-pinnate; pinnae 1-3 pairs; basal pinna ca. $0.1-0.15 \mathrm{~m}$, usually with $2-3$ pinnules; fertile fronds 1-3 pinnae; terminal pinna linear $15-20$ x $1.5 \mathrm{~cm}$. Not common.

Pteris semipinnata L.-TL 44-Tuyen Quang, Ham Yen, Yen Thuan, 22 17'30", $104^{\circ} 58^{\prime} 58^{\prime \prime}, 645 \mathrm{~m}$. In secondary wet scrubs on acidic sandstone-shale cliffs. Perennial terrestrial fern, to $0.6-0.9 \times 0.3-0.4 \mathrm{~m}$; fronds clustered, subdimorphic; stipe $0.3-0.4 \mathrm{~m}$; stipe and rachis castaneous-reddish, shiny, glabrous; lateral pinnae 5-7 pairs; fertile segments entire; basiscopic side pectinately lobed nearly to costa, acroscopic side reduced to subentire wing, base oblique, basiscopic side decurrent. Not common.

Pteris tripartita Sw.-LT 04-Tuyen Quang, Ham Yen, Yen Thuan, 22 ${ }^{\circ} 7^{\prime} 07^{\prime}$, $104^{\circ} 59^{\prime} 30^{\prime}, 608 \mathrm{~m}$. In secondary scrubs on foot of shale-sandstone cliffs. Perennial terrestrial fern, to $1.2-1.4 \mathrm{~m}$ tall; fronds clustered; stipe dark straw-colored, to $0.9 \mathrm{~m}$, glabrous; rachis dark straw, glabrous; lamina with 3,1 or 2 pinnatisect divisions, broadly ovate in outline; lateral division to $1 \mathrm{~m}$, at medium ca. $0.4-0.5 \mathrm{~m}$; pinnules $13-16$ pairs; basal pinnule usually divided into 2 or 3 forks; middle pinnules ca. $25 \mathrm{~cm}$; pinnae of 
division ca. $25 \times 6 \mathrm{~cm}$; medium pinnule with 18-26 pairs of segments, $2.5-3,5 \times 0.4-0.6$ $\mathrm{cm}$. Not common.

Pteris vittata L.-DL-BM 045- Giang, Bac Me, Minh Ngoc, 22'43'41”, 105²'12'22”, 200 $\mathrm{m}$. Rather common on open vertical cliffs, rock crevices or limestone foots. Perennial terrestrial fern, $0.7-1.2 \mathrm{~m}$ tall; rhizome $1.5-2$ $\mathrm{cm}$ in diam.; fronds clustered; stipe $9-15 \mathrm{~cm}$, straw-colored, densely scaly lower part, sparsely upward; lamina 1-pinnate, $0.4-0.8 \mathrm{x}$ $0.2-0.3 \mathrm{~m}$; lateral pinnae to $20-40$ pairs; lowermost 3-4 pairs reduced; middle pinnae 10-16 x 1.3-1.6 cm; coneosori marginal. Very common.

\section{Family Dennstaedtiaceae}

Pteridium esculentum (G.Forst.) Cokayne- LT 65- Tuyen Quang, Ham Yen, Yen Thuan, $22^{\circ} 17^{\prime} 07^{\prime \prime}, 104^{\circ} 59^{\prime} 29^{\prime \prime}, 608 \mathrm{~m}$. Perennial terrestrial fern on secondary shalesandstone grasslands. Plants ca. $0.7-1 \mathrm{~m}$ tall, lamina oblong-triangular in outline and \pm in one plane, $0.6 \times 0.3 \mathrm{~m}, 3$-pinnate-pinnatifid; pinnae 8-12 pairs; biggest pinna to $20 \times 9 \mathrm{~cm}$ with to 20 pairs of pinnules, to $7 \times 2 \mathrm{~cm}$; segments $8-10$ pairs per pinna, ca. $0.8 \times 0.5$ $\mathrm{mm}$, pinnatifid; sori near notch at margin of lobules; indusia formed by reflexed margin of lamina, interrupted. Rare.

\section{Family Aspleniaceae}

Asplenium antrophyoides H.Christ-LT 47-Tuyen Quang, Ham Yen, Yen Thuan, $22^{\circ} 17^{\prime} 07^{\prime \prime}, 104^{\circ} 59^{\prime} 29$ ', $618 \mathrm{~m}$. In secondary scrubs, on limestone or sandstone rocks. Perennial epiphytic or lithophytic; lamina narrowly oblanceolate, ca. $0.4-0.6 \mathrm{~m}$ long; midrib carinate abaxially, apex cuspidate. Not common.

Asplenium ensiforme Wall. ex Hook. \& Grev.-DL-BM 007-Ha Giang, Bac Me, Minh Ngoc, 22 43'17', 105'11'33”, $210 \mathrm{~m}$. Scattered epiphytic on tree trunks in secondary limestone woodlands. Fronds clustered; stipe $3-5 \mathrm{~cm}$ long; lamina narrowly lanceolate, ca. $0.3-0.4 \times 0.03-0.05 \mathrm{~m}$, base decurrent. Occasionally common.

Asplenium ensiforme Wall. ex Hook. \& Grev.-TL 033-Tuyen Quang Prov., Ham Yen
Distr., Phu Luu Comm., 22 12 '18", $105^{\circ} 02^{\prime} 38^{\prime \prime}, 212 \mathrm{~m}$. Epiphytic on tree trunks, in secondary scrubs of limestone stream. Young perennial fern. Rare.

Asplenium nidus L. $-L T 06 \& L T 34 \& L T$ 57-Tuyen Quang, Ham Yen, Yen Thuan, around 22 $17^{\prime} 07^{\prime \prime}, 104^{\circ} 59^{\prime} 30^{\prime \prime}, 608-659 \mathrm{~m}$. Mesophytic, heliophytic, lithophytic and epiphytic, in secondary scrubs, foot limestone and sandstone forest; fronds ca. 0.7-1.2 x 0.09-0.14m. Common. (In Asplenium nidus complex).

Asplenium oblanceolatum Copel.-DL-BM 054-Ha Giang, Bac Me, Minh Ngoc, around 22042'49”, 105'11'16", 224 m. Mesophytic, epiphytic on tree trunks or lithophytic on foot of limestone cliffs. Fronds caespitose, 0.6-0.8 $\mathrm{m}$ tall; leaves distinctly spatulate, widest above middle, ca. 7-9 cm; lamina gradually reduced into a long broadly winged basal part, apex cuspidate; sori linear, 2-3 $\mathrm{cm}$. Occasionally common. (In Asplenium nidus complex).

Asplenium oblanceolatum Copel.-TL 036-Tuyen Quang Prov., Ham Yen Distr., Phu Luu Comm., 2212'18', 10502'38', $212 \mathrm{~m}$. Epiphytic on tree trunks, in secondary scrubs of limestone stream. Young perennial fern. Rare.

Hymenasplenium obliquissimum (Hayata) Sugimono-LT 55-Tuyen Quang, Ham Yen,

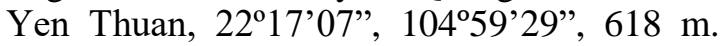
Hydrophytic and heliophytic, epiphytic, rarely lithophytic on sandstone stream banks. Perennial fern, 0.3-0.4 m tall; stipe brownish; lamina elliptic, ca. $0.2-0.3 \times 0.07-0.08 \mathrm{~m}$, thin, 1-pinnate, widest at base; 18-22 pairs of leaflets, trapeziform to falcate, $3.5-5 \mathrm{x}$ $1.2-1.5 \mathrm{~cm}$; pinnae strongly dimidiate; sori linear, 3-4 mm. Occasionally common. OK.

Family Blechnaceae

Blechnum orientale L.-TL 22-Tuyen Quang, Ham Yen, Phu Luu, 22 $12^{\prime} 38^{\prime \prime}$, $105^{\circ} 02^{\prime} 57^{\prime \prime}, 140 \mathrm{~m}$. Clustered on secondary on shale cliff scrubs. Frond blades ca. 0.6-1.2 x 0.3-0.6 m; imparipinnate; pinnae numerous, linear, 25-30 x 1-1.2 cm; sori linear, forming coenosori adjacent and parallel to costa; 
indusium attached to commissure, facing toward costa. Occasionally common.

Family Athyriaceae

Athyrium mackinnonii C.Chr.-LT 30Tuyen Quang, Ham Yen, Yen Thuan, 22017'29", 104'58'58', 659 m. Perennial, hydrophytic, terrestrial fern to $2.5-3.5 \mathrm{~m}$ tall, foot of limestone mountains; rhizome erect, 1 $\mathrm{cm}$ in diam, apex densely blackish brown scales; fronds caespitose; fertile fronds 2-2.5 $\mathrm{m}$ long; stipe $0.5-0.7 \mathrm{~m}$ long, $0.5 \mathrm{~cm}$ in diam.,densely blackish brown scales; lamina deltoid oblong, ca. $0.7-1$ x $0.5-0.9 \mathrm{~m}$; pinnae 12-18 pairs, 2-pinnate and pinnules pinnatipartite; sori and indusia horseshoeshaped.Occasionally common.

Diplazium donianum (Mett.) Tardieu-LT 24-Tuyen Quang, Ham Yen, Yen Thuan, 22¹7'29', 104'58'58', 659 m. Mesophytic perennial, terrestrial fern, on semiopened sandstone-shale cliffs. Fronds caespitose; fertile fronds $0.6-0.8 \mathrm{~m}$ tall; stipe more than $0.3 \mathrm{~m}$; lamina ovate-elliptic $0.3-0.5 \times 0.2-0.3$ m, 1-imparipinnate; lateral pinnae $3-5$ pairs, lanceolate; indusia long linear, oblique. Rare.

Diplazium esculentum (Retz.) Sw.-TL 013a- Ha Giang, Bac Me, Minh Ngoc, $22^{\circ} 12^{\prime} 36^{\prime \prime}, 105^{\circ} 03^{\prime} 29^{\prime \prime}, 233 \mathrm{~m}$. Perennial terrestrial fern in very wet foot cliffs or stream banks. Fronds $1.5 \mathrm{~m}$ tall; stipe at least $0.5 \mathrm{~m}$ long; lamina deltoid, $0.7-0.9 \times 0.3-0.4 \mathrm{~m}$, apex acuminate; $8-10$ pairs of pinnae, 2-pinnate with pinnule pinnatifid; lower pinnae broadly lanceolate; pinnules to $0.2-0.3$ x $0.06-0.08 \mathrm{~cm}$; sori linear, to $7-10$ pairs per pinnule lobes. Common.

Diplazium megaphyllum (Baker) H.ChristTL28-Tuyen Quang, Ham Yen, Phu Luu, $22^{\circ} 12^{\prime} 18^{\prime \prime}, \quad 105^{\circ} 02^{\prime} 38^{\prime}$, $212 \mathrm{~m}$. Perennial terrestrial fern in very wet limestone stream banks. Fertile fronds to $0.8-1 \mathrm{~m}$; stipes $0.3-0.4$ $\mathrm{m}$ long, densely dark brown scaly at base; lamina oblong, ca. $0.6-0.7$ x $0.4-0.5 \mathrm{~m}, 1-$ pinnate with 14-18 leaflets, ascending, base truncate-cuneate, apex attenuate to caudate; sori linear, 3-9 mm long per vein group. Rare.

Diplazium stenochlamys C.Chr.-TL 016Tuyen Quang, Ham Yen, Phu Lưu, 22¹2'36”,
10503'29”, 233 m. Perennial terrestrial fern in very wet limestone cliffs. Fertile fronds ca. $1.5 \mathrm{~m}$ long; stipe at least $0.5 \mathrm{~m}$ long, glabrous; lamina glabrous, papery, deltoid, 0.7-0.9 x 0.3-0.4 m, apex acuminate; 8-10 pairs of pinnae, alterne, ascending; basal 1 or 2 pinnae oblong, 20-25 × 6-8 cm, pinnatilobate to costa; sori linear, to $7-10$ pairs per pinnule lobes. Rare.

Family Thelypteridaceae

Christella balansae (Ching) Holttum-TL 031-Tuyen Quang, Ham Yen, Phu Luu, $22^{\circ} 12^{\prime} 38^{\prime},, 105^{\circ} 02^{\prime} 57^{\prime \prime}, 140 \mathrm{~m}$. Edges of very wet limestone stream banks. Perennial terrestrial fern, 0.8-1.2 m; stipe $0.15-0.25 \mathrm{~m}$; laminae oblong-lanceolate, $0.7-0.9 \mathrm{~m}$; lateral pinnae 20-25 pairs, proximal 1-4 abruptly shortened, triangular-auriculate, $1-2 \mathrm{~cm}$, reflexed; middle pinnae lanceolate, 10-14 x 1.5-2 cm, base truncate, lobed ca. 1/4 toward costae; sori orbicular. Occasionally common.

Family Thelypteridaceae

Cyclosorus parasiticus (L.) Farw.-DL-BM 042-Ha Giang, Bac Me, Minh Ngoc, 22 43 '41', 105'12'22”, $200 \mathrm{~m}$. In open, wet secondary grasslands and scrubs. Perennial terrestrial fern, $0.7-1.2 \mathrm{~m}$ tall; rhizomes with dark brown scales; laminae with thin acicular hairs throughout on both surface, 0.4-0.6 x $0.15-0.25 \mathrm{~m} ; 14-22$ pairs, lobed $1 / 2-3 / 4$ toward costae; proximal 1 or 2 pairs reflexed, not reduced; middle pairs $8-12$ x $1.2-1.8 \mathrm{~cm}$; sori orbicular. Common.

Cyclosorus truncatus (Poir.) Farw.- DLBM 004 \& DL-BM 061-Ha Giang, Bac Me, Minh Ngoc, around 22\%43'17', 105'11'33', $210 \mathrm{~m}$. Open wet secondary scrubs along sandstone-shale stream banks. Ferns $1.2-1.8 \mathrm{~m}$ tall; stipes $0.6-0.8 \mathrm{~m}$, with brown scales; laminae oblong-lanceolate in outline, $0.9-1.3 \mathrm{x}$ 0.3-0.4 m; pinnae 32-40 pairs; $3-6$ proximal pairs abruptly shortened; middle pinnae $21-26$ x $2-2.5 \mathrm{~cm}$, lobed $1 / 3-1 / 2$ toward costae; sori orbicular. Occasionally common.

Cyclosorus truncatus (Poir.) Farw.-LT 19TuyenQuang, Ham Yen, Yen Thuan, 22 $2^{\circ} 17^{\prime} 29^{\prime \prime}, 104^{\circ} 58^{\prime} 58^{\prime \prime}, 659$ m. Open rather wet sandstone scrubs on foot of limestone 
mountains. Habit the same as $D L-B M 004 \&$ $D L-B M$ 061. Not rare.

Pronephrium hirsutum Ching ex Y.X.LinTL 042-Tuyen Quang Prov., Ham Yen Distr., Phu Lưu, 22 $12^{\prime} 10^{\prime \prime}, 105^{\circ} 02^{\prime} 40^{\prime \prime}, 152 \mathrm{~m}$. Scattered on secondary shale cliff scrubs. Perennial terrestrial fern, $1 \mathrm{~m}$ tall; rhizomes long creeping with sparse brown scales; stipes 0.4-0.5 m; laminae ovate-lanceolate in outline, 1-imparipinnate; lateral pinnae 3-4 pairs; pinnae $15-20 \times 3-4.5 \mathrm{~cm}$; terminal pinna ca. $25 \times 5-6 \mathrm{~cm}$; sori orbicular, not confluent when mature, exindusiate. Rare.

Pronephrium simplex var. trifoliata (Ching) Loc K. Phan, var. nov. (basionym: Abacopteris simplex var. trifoliata Ching)-TL 031-Tuyen Quang Prov., Ham Yen Distr., Phu Lưu, $22^{\circ} 12^{\prime} 50^{\prime \prime}, 105^{\circ} 02^{\prime} 30^{\prime \prime}, 125 \mathrm{~m}$. Scattered in open wet shale cliffs. Perennial terrestrial mesophytic, $0.4-0.5 \mathrm{~m}$ tall; fronds dimorphic; laminae ovate-triangular in outline, ternate; lateral leaflets of fertile fronds 9-11.5 x 1.5 $\mathrm{cm}$; terminal leaflet $15 \times 1.5 \mathrm{~cm}$; sori orbicular, becoming confluents when old, exsindusiate; sterile leaflets shorter and larger. Rather common. Notes: Some pteridologists consider that this taxon is Abacopteris simplex var. trifoliata Ching, Cyclosorus simplex var. trifoliata (Ching) T.J.Liu, or Thelypteris simplex var. trifoliata (Ching) C.F.Reed.

\section{Family Dryopteridaceae}

Bolbitis christensenii (Ching) Ching-TL 034-Tuyen Quang, Ham Yen, Phù Lưu, $22^{\circ} 12^{\prime} 18^{\prime \prime}, 105^{\circ} 02^{\prime} 38^{\prime \prime}, 212 \mathrm{~m}$. Rhizome $1 \mathrm{~cm}$ in diam., densely brownish black scales. Fronds tufted; sterile fronds pinnate with lamina elliptic in outline, $0.4-0.5 \times 0.25 \mathrm{~m}$, pinnae 7-11 pairs, 9-12 x 1.8-2.4 cm; fertile fronds as long as sterile fronds but lamina obviously narrower; stipe $0.4 \mathrm{~m}$; blade ca. $0.3-0.35 \mathrm{~m}, 8-10$ pairs of pinnae, lanceolate, $3-5 \times 0.7-1 \mathrm{~cm}$. Sori acrostichoid abaxial face without indusia. Rare.

Bolbitis heteroclita (C.Presl) Ching-LT 36, LT 53 \& LT 61-Tuyen Quang, Ham Yen, Yen Thuan, around 22 $17^{\prime} 29^{\prime \prime}, 104^{\circ} 58^{\prime} 58^{\prime \prime}$, 608-659 m. Mesophytic edge of sandstone cave. Rhizome robust, long creeping, densely brownish black scaly. Fronds clustered. Sterile fronds with stipes $14-21 \mathrm{~cm}$ and lamina $15-50$ × $8 \mathrm{~cm}$, pinnae 3-6 pairs; apical with up to 5-11 pairs of lobes, apex caudate to long flagelliform, terminal part with bulbil; fertile fronds $0.3-0.4 \mathrm{~m}$ long; stipes ca. $0.2 \mathrm{~m}$, densely scaly; pinnae 4-7 pairs; apical part 7-11 x 2-3.5 cm; Sori acrostichoid abaxial face. Not rare.

Dryopteris varia (L.) Kunze-LT 028Tuyen Quang, Ham Yen, Yen Thuan, $22^{\circ} 17^{\prime} 30^{\prime \prime}, 104^{\circ} 58^{\prime} 58^{\prime \prime}, 645 \mathrm{~m}$. In mesophytic secondary sandstone-shale cliff scrubs. Perennial terrestrial fern, $0.5-0.7 \mathrm{~m}$ tall, dimorphic; fertile fronds up to $0.6 \mathrm{~m}$ tall; stipes covered with scales same as on rhizome; lamina $0.3-0.45 \times 0.24-0.28 \mathrm{~m}$, tripinnatifid with pinnae 15-19 pairs; basal pair largest, ca. 15 x $10 \mathrm{~cm}$, basiscopic longest, ca. 6 x $2 \mathrm{~cm}$, ultimate pinnules acuminate; rachis and pinna rachis covered with brown scales; sori middle between margin and costa; indusia reniform. Rare.

Family Nephrolepidaceae

Nephrolepisbiserrata (Sw.) Schott-TL 059-Tuyen Quang, Ham Yen, Phu Luu, around 22 $2^{\circ} 12^{\prime} 36^{\prime \prime}, 105^{\circ} 03^{\prime} 22^{\prime \prime}, 54-163 \mathrm{~m}$. Densely epiphytic, xerophytic in axil leaf of Livistona spp. Fronds dimorphic; fertile laminae glabrous on both surfaces, 0.6-1.8 x $0.2 \mathrm{~m}$ with $50-60$ pairs of pinnae, $2-15 \mathrm{x}$ $0.17-2.2 \mathrm{~cm}$, base not auriculate on acroscopic side; sori orbicular, closer to margin than to midvein.CommonNephrolepidaceae. Nephrolepis biserrata (Sw.) Schott- TL 013-Tuyen Quang, Ham Yen, Phu Luu, around 22 $12^{\prime} 36^{\prime \prime}, 105^{\circ} 03^{\prime} 22^{\prime \prime}$, $163 \mathrm{~m}$. In groups on mesophytic secondary shale cliff scrubs. Habit same as TL 059. Rare.

Nephrolepis cordifolia (L.) C.Presl.-TB 130-Tuyen Quang, Ham Yen, Yen Thuan,

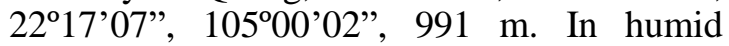
valley disturbed scrubs. Perennial lithophytic fern, stipe 4-7 cm, densely covered by yellowish-brown scales, scattered on rachis; lamina base strongly reduced, narrowly lanceolate, $0.3-0.5 \mathrm{~m}$ long, $3-4 \mathrm{~cm}$ wide at 
middle pinnate; pinnae 45-60 pairs, unequal at base, auricle acute; sori orbicular-reniform; indusia suborbicular. Occasionally common.

\section{Family Tectariaceae}

Arthropteris palisotii (Desv.) Alston-LT 54-Tuyen Quang, Ham Yen, Yen Thuan, $22^{\circ} 17^{\prime} 07^{\prime \prime}, 104^{\circ} 59^{\prime} 30$ ", $608 \mathrm{~m}$. In secondary evergreen scrubs, on wet sandstone stream banks. Perennial epiphytic or lithophytic fern; rhizome creeping, 2-3 m long or more; fronds $3-10 \mathrm{~cm}$ apart; lamina lanceolate, 10-21 x 2-4cm; pinnae $15-20$ pairs, downward ones reduced and deflexed; middle ones $2-4$ x $0.6-0.9 \mathrm{~cm}$, auriculate on upper side, apex rounded; sori very near margin. Occasionally common.

Pleocnemia winitii Holttum-TL 002,TL 012\& TL 043-Tuyen Quang Prov., Ham Yen Distr., Phu Lưu, around $22^{\circ} 12^{\prime} 38^{\prime \prime}$, $105^{\circ} 02^{\prime} 58^{\prime \prime}, 54-125 \mathrm{~m}$. Scattered in wet scrubs on shale cliffs, to 1-2 m tall. Rhizome with dark brown scales same as on stipe; lamina $1.5 \times 1-1.2 \mathrm{~m}, 3$-or 4-pinnatifid; basal pinnae largest, ca. $0.3-0.4$ x $0.23-0.3$ $\mathrm{m}$; basal basiscopic primary pinnule elongate, ca. $0.25-0.3 \mathrm{~m}$; indusia usually absent. Rather common.

Tectaria decurrens (C.Presl) Copel.-TL 14 \& TL 57-Tuyen Quang Prov., Ham Yen Distr., Phu Lưu, around $22^{\circ} 12^{\prime} 02^{\prime \prime}, 105^{\circ} 02^{\prime} 15^{\prime \prime}$, 125-143 m. Scattered in wet scrubs on shale cliffs. Perennial terrestrial fern, $0.7-1.2 \mathrm{~m}$ high; fronds clustered, dimorphic; stipes 0.4-0.6 m, winged nearly to base; fertile lamina $0.6-0.9 \times 0.3-0.6 \mathrm{~m}$, deeply pinnately incised; lateral pinnae 3-4 pairs, ascending; sori large, in two rows between adjacent main veins. Rather common.

Tectaria devexa (Kunze) Copel.-DL-BM 031 \& DL-BM 039- Ha Giang, Bac Me, Minh Ngoc, around 22\%43'41', 105'12'22”, 200-224 $\mathrm{m}$. Very common on open mesophytic sandstone-shale vertical cliffs. Perennial terrestrial fern, ca. $0.4-0.7 \mathrm{~m}$ tall; fronds clustered; stipe $0.2-0.3 \mathrm{~m}$ long; lamina triangular, $0.25-0.4 \times 0.2-0.3 \mathrm{~m}$, tripinnatepinnatifid, bipinnatifid toward apex; pinnae
3-5 pairs; basal pinnae largest, with somes subseparate basiscopic pinnules; sori orbicular; indusia orbicular-reniform. Common.

Tectaria devexa (Kunze) Copel.-LT 35Tuyen Quang, Ham Yen, Yen Thuan, $22^{\circ} 17^{\prime} 29^{\prime \prime}, 104^{\circ} 58^{\prime} 58^{\prime \prime}, 659 \mathrm{~m}$. Habitat and habit same as $D L-B M 031 \& D L-B M 039$.

Tectaria fuscipes (Wall. ex Bedd.) C.Chr.LT 26- Tuyên Quang, Hàm Yên, Yên Thuận, $22^{\circ} 17^{\prime} 29$ ", $104^{\circ} 58^{\prime} 58^{\prime \prime}, 659 \mathrm{~m}$. In wet sandstone-shale scrubs. Terrestrial perennial fern, ca $0.5-0.6 \mathrm{~m}$ tall; rhizomes and stipe base densely brownish scales; base fronds dimorphic; sterile fronds with stipes $0.3-0.4$ $\mathrm{m}$ and lamina $18-26 \times 11-15 \mathrm{~cm}$; lamina of fertile fronds ovate-triangular, 15-22 x 7-14 $\mathrm{cm}$; pinnae 1-2 pairs; basal pair largest, ca. 8-11 x 3.5-4.5 cm, asymmetric, margin deeply lobed without free pinnules, pinnatipartite to $1 / 2-3 / 4$ way toward costa; sori orbicular, in 2 irregular rows between main vein pairs for each lobe. Rare.

Tectaria kusukusensis (Hayata) Lellinger$D L-B M$ 048-Ha Giang, Bac Me, Minh Ngoc, $22^{\circ} 42^{\prime} 49^{\prime \prime}, 105^{\circ} 11^{\prime} 16$ ", $224 \mathrm{~m}$. Epiphytic on trees, rarely on rocks crevices along open stream banks. Perennial fern, 0.7-0.9 m tall; stipe $0.4-0.5 \mathrm{~m}$ long, base densely dark brown scaly; fronds subdimorphic; lamina bipinnatifid to tripinnatifid, oblong, $0.4-0.6 \mathrm{x}$ $0.25-0.3 \mathrm{~m}$; pinnae $8-10$ pairs; basal pair obliquely triangular, $17-20$ x $6-8 \mathrm{~cm}$; middle pinnae $9-14 \times 2.5-3.5 \mathrm{~cm}$, pinnatipartite to 4/5 toward costa; sori orbicular, 3-8 pairs, regular 1 row beside main veins. Rare.

Tectaria phaeocaulis (Rosenst.) C.Chr.LT 21-Tuyen Quang, Ham Yen, Yen Thuan, $22^{\circ} 17^{\prime} 29^{\prime \prime}, 104^{\circ} 58^{\prime} 58^{\prime \prime}, 659 \mathrm{~m}$. In sandstoneshale cliff scrubs. Perennial terrestrial mesophytic fern, ca. $0.8-1 \mathrm{~m}$ tall, slightly dimorphic; rhizomes and stipe base scattered brown-black scales; fertile fronds with stipe to $0.5 \mathrm{~m}$ long; lamina long triangular, ca. $0.5 \mathrm{x}$ $0.3 \mathrm{~m}$, pinnae $4-5$ pairs; basal pinna bipinnate to tripinnatifid, $0.3 \times 0.25 \mathrm{~m}$ with stalk $1-2$ $\mathrm{cm}$; middle pinnae 20-27 x 4-5 cm; sori large, orbicular, regularly in 2 rows. Occasionally common. 
Tectaria quinquefida (Baker) Ching-DL$B M$ 065-Ha Giang, Bac Me, Minh Ngoc, $22^{\circ} 42^{\prime} 49^{\prime \prime}, 105^{\circ} 11^{\prime} 16^{\prime \prime}, 224 \mathrm{~m}$. Growing on very humid stream limestone banks. Perennial terrestrial ombrophylous fern, $0.6-0.8 \mathrm{~m}$ tall; rhizomes and base of stipes densely dark brown scales; fronds subdimorphic; fertile fronds deltoid-ovate, ca. $0.3 \times 0.2 \mathrm{~m}$; terminal pinna trilobed; lowest pinna bifide; sori small, in irregular rows. Rare.

Tectaria sagenioides (Mett.) Christenh.$L T$ 44-Tuyen Quang, Ham Yen, Yen Thuan, $22^{\circ} 17^{\prime} 07^{\prime \prime}, 104^{\circ} 59^{\prime} 29^{\prime \prime} .618 \mathrm{~m}$. In open, wet shale-sandstone scrubs. Perennial terrestrial fern, $0.7-0.8 \mathrm{~m}$ tall, rhizome to $1.8 \mathrm{~cm}$ in diam., densely blackish brown scaly at apex and base of stipe; fronds monomorphic, clustered; stipe $0.2-0.3 \mathrm{~m}$ long; lamina elliptic-triangular, $0.5-0.6 \times 0.2-0.25 \mathrm{~m}$; lateral pinnae $8-10$ pairs; basal pinna slightly shortened, middle pinnae $12-15 \times 2.5-3.5 \mathrm{~cm}$, pinnatipartite to $2 / 3$; lobes with 14 pairs of sori, exindusiate. Rare.

Family Tectariaceae

Tectaria simonsii (Baker) Ching - DL-BM 049-Ha Giang, Bac Me, Minh Ngoc, 22042'49", 105'11'16”, 224 m. Epiphytic, rarely on limestone crevices along open stream banks. Terrestrial perennial fern, $0.7-0.9 \mathrm{~m}$ tall; rhizome to $1.5 \mathrm{~cm}$ in diam.; fronds subdimorphic; stipe rachis dark brown; lamina bipinnatifid to tripinnatifid, subpentagonal, $0.4-0.6 \times 0.25-0.3 \mathrm{~m}$; pinnae 4-6 pairs; basal pair largest; sori orbicular, in \pm regular rows. Rare.

Tectaria subsageniacea (H.Christ) Christenh.-LT 43-Tuyen Quang, Ham Yen, Yen Thuan, around $22^{\circ} 17^{\prime} 07^{\prime \prime}, 104^{\circ} 59^{\prime} 29^{\prime \prime}$, $618 \mathrm{~m}$. In wet grasslands along limestone stream banks. Perennial terrestrial fern, 1.4$1.8 \mathrm{~m}$ tall; stipe $0.5-0.7 \mathrm{~m}$ long, densely black brown setae; fronds clustered; lamina $0.8-1 \times 0.3-0.4 \mathrm{~m}$; rachises deep brown with scarcely scales and hairs; pinnae 18-22 pairs; no shortened and deflexed distal pinnae; middle pinnae ca. $20 \times 4 \mathrm{~cm}$, pinnatipartite to $2 / 3-3 / 4$; sori orbicular, small, exindusiate. Rare.
Family Oleandraceae

Oleandra wallichii (Hook.) C.Presl-LT 13-Tuyen Quang, Ham Yen, Yen Thuan,

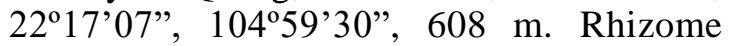
long creeping, freely branching, 3-4 $\mathrm{mm}$ in diam.; fronds in small tuffs on tops; petiole $1 \mathrm{~cm}$ long; lamina narrowly oblong, 12-18 $\mathrm{x} 1.1-2.4 \mathrm{~cm}$, both surfaces hairy, base cuneate, apex apiculate-caudate; sori very close to costa; indusia reniform. Very common.

\section{Family Davalliaceae}

Davallia divaricata Blume-LT 11-Tuyen Quang, Ham Yen, Yen Thuan, 22 $17^{\prime} 07^{\prime \prime}$, 104'59'30”, 608 m. Perennial xerophytic epiphytic, on leaf axil of Livistona spp. Rhizome $0.8-1.5 \mathrm{~cm}$ in diam., soft, densely covered with brownish scales; stipes $10-21 \mathrm{~cm}$; lamina narrow triangular, $0.4-0.7 \times 0.2-0.35$ $\mathrm{m}$, tripinnate toward base; pinnae $6-9$ pairs, to $20 \times 6 \mathrm{~cm}$; pinnules ca. 20 pairs; pinnules of at least larger pinnae anadromous, deltoid; sori separate, borne several on a segment. Very common.

Davallia divaricata Blume-TLD 034Tuyen Quang, Ham Yen, Phù Lưu, Nậm Lương, around point $22^{\circ} 12^{\prime} 45^{\prime \prime}, 105^{\circ} 04^{\prime} 47^{\prime \prime}$, $970 \mathrm{~m}$. Epiphytic in wet woodlands, foot of Cham Chu shale mountain. Habit same as $L T$ 11. Rather common. OK.

Davallia solida (G.Forst.) Sw.-LT 10Tuyen Quang, Ham Yen, Yen Thuan, 22 ${ }^{\circ} 17^{\prime} 07^{\prime},, 104^{\circ} 59^{\prime} 30^{\prime \prime}, 608 \mathrm{~m}$. Perennial xerophytic epiphytic, on leaf axil of Livistona spp. Rhizome $0.4-0.6 \mathrm{~cm}$ in diam., soft, densely covered with brownish scales; stipes 8-12 cm; lamina triangular in outline, ca. $0.12-0.18 \times 0.10-0.14 \mathrm{~m}$, bipinnate or tripinnate toward base; longest pinnae ca. $15 \mathrm{x}$ $7 \mathrm{~cm}$; sori separate, borne several on a segment. Very common.

Family Polypodiaceae

Aglaomorpha coronans (Wall. ex. Mett.) Copel.-LT 09-Tuyen Quang, Ham Yen, Yen Thuan, 22 ${ }^{\circ} 17^{\prime} 07^{\prime \prime}, 104^{\circ} 59^{\prime} 30^{\prime \prime}, 608 \mathrm{~m}$. Perennial xerophytic, epiphytic, rarely lithophytic fern in disturbed woodlands; rhizome ca. $2-3 \mathrm{~cm}$ in diam.; fronds 
monomorphic, glabrous, $0.7-1.2 \times 0.2-0.3$ $\mathrm{m}$, with dilated base ca. $18 \times 15 \mathrm{~cm}$; pinnae 11-22 pairs; middle pinnae ca. $18 \times 3 \mathrm{~cm}$, margin entire, apex attenuate; sori in several rows between midrib and margin, one in each main areole, slightly sunken. Occasionally common.

Drynaria bonii H.Christ- DL-BM 010- Ha Giang, Bac Me, Minh Ngoc, 22 43'17', $105^{\circ} 11^{\prime} 33^{\prime \prime}, 210 \mathrm{~m}$. Scattered epiphytic on tree trunks in sligthly disturbed evergreen broad-leaved on foot of limestone. Rhizome shortly creeping, dorsiventrally flattened; fronds dimorphic, glabrous; basal frond completely covering and surrounding rhizome, sessile, $8-10 \times 5-8 \mathrm{~cm}$; foliage fronds stalked; stipe ca. 15-22 cm long; lamina pinnatifid up to ca $1 \mathrm{~mm}$ from costa, $0.5-0.7 \times 0.2-0.3 \mathrm{~m}$, apex aborted; pinnae 4-6 pairs, broadly lanceolate, 7-18 x 2.5-3.5 $\mathrm{cm}$; sori in 2 irregular rows between lateral veins. Occasionally common.

Drynaria bonii H.Christ-TL 035-Tuyen Quang, Ham Yen, Phu Luu, 22 $12^{\prime} 18^{\prime}$, $105^{\circ} 02^{\prime} 38^{\prime \prime}, 212 \mathrm{~m}$. Rhizomes cling tightly on foot of treelet in the fast flowing water, in heavily destroyed broad-leaved forest. Habit same as $D L-B M$ 010. Rare.

Lepidomicrosorium superficiale (Blume) L.Wang- $D L-B M$ 006-Tuyen Quang, Ham Yen, Yen Thuan, 22 ${ }^{\circ} 17^{\prime} 07^{\prime \prime}, 104^{\circ} 59^{\prime} 29 ”, 618$ $\mathrm{m}$. In secondary wet shale-sandstone grasslands along stream. Perennial epiphytic, rarely lithophytic fern; rhizome $2-3 \mathrm{~mm}$ in diam.; fronds monomorphic, $0.4-0.5 \mathrm{x}$ 0.04-0.05 m; lamina simple, elliptic, base decurrent along stipe dark brown, apex acute; sori separate, forming 2 regular rows between each pair of lateral veins, orbicular, ca. $2 \mathrm{~mm}$ in diam., superficial. Occasionally common.

Lepidomicrosorium superficiale (Blume) L.Wang-LT 52- Tuyen Quang, Ham Yen, Yen

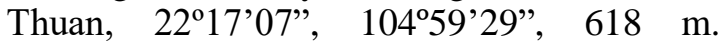
Habitat and habit similar to $D L-B M 006$. Common.

Leptochilus ellipticus (Thunb.) Noot.-LT 38-Tuyen Quang, Ham Yen, Yen Thuan, $22^{\circ} 17^{\prime} 29^{\prime \prime}, 104^{\circ} 58^{\prime} 58^{\prime \prime}, 659 \mathrm{~m}$. On edges of mesophytic shale-sandstone cave. Perennial fern; rhizome long creeping; scale dark brown; fronds subdimorphic; stipes brownish, 10-22 cm long, winged to $0.3 \mathrm{~cm}$ wide; sterile lamina pinnate to deeply pinnatifid; fertile lamina ca. $10-14 \mathrm{~cm}$ with stipes $15-15 \mathrm{~cm}$ long; pinnae 5-7 pairs, ca. $3-5 \times 0.2-0.5 \mathrm{~cm}$; sori linear, one between each pair of lateral veins. Rare.

Leptochilus henryi (Baker) X.C.Zhang-LT 25-Tuyen Quang, Ham Yen, Yen Thuan, $22^{\circ} 17^{\prime} 29^{\prime \prime}, 104^{\circ} 58^{\prime} 58^{\prime \prime}, 659 \mathrm{~m}$. On edges of mesophytic shale-sandstone scrubs. Perennial epiphytic or lithophytic fern. Rhizome slender, long creeping; fronds monomorphic; stipe 2-4 cm; lamina oblong-lanceolate, 0.3-0.4 x 0.04-0.05 m, glabrous, base narrowed into narrow wing along stipe, apex obtus; sori linear, 1 regular row between lateral veins. Rare.

Microsorum membranaceum (D.Don) Ching-LT 58-Tuyen Quang, Ham Yen, Yen Thuan, 22 ${ }^{\circ} 17^{\prime} 07^{\prime \prime}, 104^{\circ} 59^{\prime} 29^{\prime \prime}, 618$ m. In secondary wet foot of limestone grasslands. Perrenial fern, epiphytic or lithophytic, 0.7-1 $\mathrm{m}$; rhizome creeping; fronds scattered along rhizome; stipes $8-15 \mathrm{~cm}$; fronds monomorphic, membranous, base narrowly decrescent, winged almost to base; lamina elliptic--oblanceolate, $0.8-0.9 \times 0.08-0.09 \mathrm{~m}$, slightly translucent when dry;sori separate on whole surface; fronds sometimes are shed seasonally. Not rare.

Microsorum punctatum (L.) Copel.-LT 02, LT $03 \& L T$ 22-Tuyen Quang, Ham Yen, Yen Thuan, around, 22\%17'29", 104'58'58', 608-659 m. In secondary mesophytic sandstone-shale scrubs. Epiphytic, lithophytic or terrestrial; rhizome shortly creeping, thick; fronds monomorphic; lamina oblanceolate, $0.4-0.6 \times 0.06-0.08 \mathrm{~m}$, soft, base narrowly decrescent, stipe winged for a considerable part; midrib glabrous; apex acute to acuminate; sori small, mostly irregularly scattered or in 8 irregular rows between costa and margin. Not rare.

Microsorum punctatum (L.) Copel.-DLBM 072-Ha Giang, Bac Me, Minh Ngoc, 
22 43'17”, $105^{\circ} 11^{\prime} 33^{\prime \prime}, 210 \mathrm{~m}$. Habitat and habit same as LT 02, LT $03 \& L T 22$. Not rare.

Neolepisorus zippelii (Blume) L.WangLT 52-Tuyen Quang, Ham Yen, Yen Thuan, $22^{\circ} 17^{\prime} 07^{\prime \prime}, 104^{\circ} 59^{\prime} 30^{\prime \prime}, 608 \mathrm{~m}$. In sandstone scrubs along stream banks. Perennial epiphytic fern. Occasionally common.

Neolepisorus minor W.M.Chu- DL-BM 070- Ha Giang, Bac Me, Minh Ngoc, $22^{\circ} 42^{\prime} 49^{\prime \prime}, 105^{\circ} 11^{\prime} 16$ ", $224 \mathrm{~m}$. In wet scrubs along limestone stream banks. Epiphytic. Rhizomes long creeping; fronds distant; stipe slender, 3-8 cm long; lamina simple, elliptic, thin papery, base long decurrent till base of stipe, apex acuminate; midrib prominent; lateral veins up to 25 pairs, obvious. Rare.

Neolepisorus ovatus (Wall. ex Bedd.) Ching-DL-BM 071-Ha Giang, Bac Me, Minh Ngoc, 22 42'49', 105'11'16", $224 \mathrm{~m}$. Growing on limestone stream rocky banks; rhizome long creeping; fronds distant, all plant parts glabrous; stipes $8-18 \mathrm{~cm}$; lamina ovate-triangular, ca. $10-15 \times 5-6 \mathrm{~cm}$, base cuneate to decurrent, apex acute; texture papery; midrib prominent; lateral veins up to 20-30 pairs, obvious. This is f. ovatus of variable species. Rare.

Phymatosorus cuspidatus (D.Don) Pic.Serm.-DL-BM 001-Ha Giang, Bac Me,

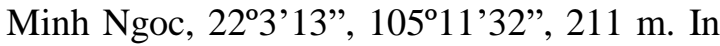
rock holes of limestone mountains. Perennial herbaceous fern; Frondsto $0.8-1 \mathrm{~m}$ tall; stipe dark brown; lamina imparipinnate, up to $0.6 \mathrm{x}$ $0.4 \mathrm{~m}$; lateral pinnae 11-14 pairs, \pm ascending, $15-28 \times 0.5-0.35 \mathrm{~cm}$; sori orbicular, in 1 row on each side of costa. Rare.

Pyrrosia porosa (C.Presl) Hovenkamp-LT 12-Tuyen Quang Prov., Ham Yen Distr., Yen Thuan, 22 ${ }^{\circ} 17^{\prime} 07^{\prime \prime}, 104^{\circ} 59^{\prime} 30^{\prime}$, 608 m. Plant xerophytic, epiphytic in leaf axil of Livistona spp., foot of sandstone mountains. Fronds subdimorphic; rhizome elongated; sterile fronds up to $0.1-0.2 \mathrm{~m}$ tall; lamina fertile fronds lanceolate, ca. $10 \times 1.5 \mathrm{~cm}$, base narrowly narrowed, apex acute; sori superficial. Rare.

\section{CONCLUSION}

The pteridophyte flora on Cham Chu mountain and on somes of its adjacent sites was studied for the first time. 123 collected numbers was gathered during 3 fielworks in 2018-2019. They are arranged in descending sequence of principal ranks (Shenzhen Code, 2018). According to PPI (2016) they belong to 84 species in 43 genera and 23 families. The $1^{\text {st }}$ class Lycopodiopsida comprises 2 orders Lycopodiales with 1 family, 1 genus and 3 species and Selaginellales with 1 family, 1 genus and5 species. The $2^{\text {nd }}$ class Polypodiopsida comprises 7 orders, 21 families, 41 genera and 76 species. The richest in number of genera and species family is Polypodiaceae with 8 genera and 12 species. The richest in number of species genera are Pteris with 8 species and Tectaria with 9 species. All presented data on pteridophyte flora of Ha Giang and Tuyen Quang Provinces are published for the first time. As cited in The NEF Bio-ecological Nature Conservation Project, this study would be enlarged until 2021 in more areas of Mountainous Region of North Vietnam.

Acknowledgements: The present study, including samples used in the thesis of student Nguyen Cong Toan (2019) was supported by The NEF Bio-ecological Nature Conservation Project in Mountainous Region of North Vietnam.

\section{REFERENCES}

DeVol C. E., Kuo C. M., Shieh W. C., Shier W. C., Tsai J. L., Yang T. Y., 1994. Flora of Taiwan, 2nd edition Vol. 1. Pteridophyta, Gymnospermae. Editorial Committee of the Flora of Taiwan Taipei, Taiwan, pp. 23-559.

Parris B. S., Kiew R., Chung R. C. K., Saw L. G., 2013. Flora of Peninsular Malaysia Series I: Ferns and Lycophytes, Vol. 2. Malayan Forest Records No 48. Ministry of Science, Technology and Innovation, Government of Malaysia, $241 \mathrm{pp}$. 
Parris B. S., Kiew R., Chung R. C. K., Saw L. G., Soepadmo E., 2010. Flora of Peninsular Malaysia. Series I: Ferns and Lycophytes, Vol. 1. Malayan Forest Records No 48 Ministry of Science, Technology and Innovation, Government of Malaysia, $249 \mathrm{pp}$.

PPG I (2016), 2016. A community-derived classification for extant lycophytes and ferns. JSE, 54(6): 563-603.

Pham Hoang Ho, 1991. An Illustrated Flora of Vietnam. Vol. I, part 1: 18-261. Montreal. Published by the author.

Phan Ke Loc, 2010. The Updated Checklist of the Fern Flora of Vietnam following the classification Scheme of A. Smith et al. (2006). J Fairylake Bot Gard,9(3): 1-13.

Shenzhen Code 2018, 2018. International Code of Nomenclature for algae, fungi, and plants (Shenzhen Code) adopted by the Nineteenth International Botanical Congress Shenzhen, China, July 2017. Koeltz Botanical Books, 254 pp.

Tagawa M., Iwatsuki K., 1979. Flora of Thailand. Vol. 3, parts 1. Pteridophytes. The Forest Herbarium, National Park, Wildlife and Plant Conservation Department, Bangkok, 129 pp.

Tagawa M., Iwatsuki K., 1985. Flora of Thailand. Vol. 3, parts 2. Pteridophytes.
The Forest Herbarium, National Park, Wildlife and Plant Conservation Department, Bangkok, 168 pp.

Tagawa M., Iwatsuki K., 1988. Flora of Thailand. Vol. 3, parts 3. Pteridophytes. The Forest Herbarium, National Park, Wildlife and Plant Conservation Department. Bangkok, 184 pp.

Tagawa M., Iwatsuki K., 1989. Flora of Thailand. Vol. 3, parts 4. Pteridophytes. The Forest Herbarium, National Park, Wildlife and Plant Conservation Department. Bangkok, 159 pp.

Tardieu-Blot M. L., Christensen C., 19391951. Fl. Gen. Indo-China, Vol. 7, part 2. Vascular Cryptogam.: 1-546 and Alston A.: 546-600, Paris. Masson \& Cie Ed. [in French]

Wu Z. Y., Raven P. H., Hong D. Y., 2013a. Flora of China- Lycopodiaceae through Polypodiaceae. 2-3. Science Press (Beijing) \& Missouri Botanical Garden Press (St. Louis), 960 pp.

Wu Z. Y., Raven P. H., Hong D. Y., 2013b. Flora of China IllustrationsLycopodiaceae through Polypodiaceae. 2-3. Science Press (Beijing) \& Missouri Botanical Garden Press (St. Louis), $960 \mathrm{pp}$. 